\title{
Impaired lipophagy in endothelial cells with prolonged exposure to oxidized low-density lipoprotein
}

\author{
CAI-PING ZHANG ${ }^{1 *}$, XIN-XIN DING $^{12^{*}}$, TIAN TIAN ${ }^{3 *}$, BO-JIE LI ${ }^{1}$, CHU-YAO WANG $^{1}$, SU-SU JIANG ${ }^{1}$, \\ JIN-QI SHAO ${ }^{1}$, YU-LIN YUAN ${ }^{1}$, YING TIAN $^{1}$, MIN ZHANG $^{1}$ and SHI-YIN LONG ${ }^{1}$ \\ ${ }^{1}$ Department of Biochemistry and Molecular Biology, Medical College, University of South China, Hengyang, \\ Hunan 421001; ${ }^{2}$ Department of Pathology, The Second People Hospital of Dezhou, Dezhou, Shandong 253000; \\ ${ }^{3}$ Department of Clinical Laboratory, The First Hospital of Changsha, Changsha, Hunan 410005, P.R. China
}

Received October 30, 2019; Accepted May 27, 2020

DOI: $10.3892 / \mathrm{mmr} .2020 .11345$

\begin{abstract}
Oxidative stress induces the formation of oxidized low-density lipoprotein (ox-LDL), which accelerates the development of atherosclerosis and the rupture of atherosclerotic plaques by promoting lipid accumulation and inhibiting autophagy in vascular cells. Lipophagy is known to be involved in maintaining the balance of neutral lipid metabolism; however, the phenomenon of lipophagy deficiency in ox-LDL-treated endothelial cells (ECs) remains to be elucidated. It has been demonstrated that lipid accumulation caused by ox-LDL inhibits autophagy, which promotes apoptosis in ECs. The aim of the present study was to investigate the association between decreased autophagy and lipid accumulation in ECs treated with ox-LDL. Electron microscopy demonstrated that the formation of autolipophagosomes was decreased in ox-LDL-treated human umbilical vein ECs compared with that in the LDL-treated group and was accompanied by a decrease in the autophagy-associated proteins via western blotting analysis. Using laser focal colocalization detection, decreased lipid processing was observed in the lysosomes of ox-LDL-treated ECs, which indicated that lipophagy may be
\end{abstract}

Correspondence to: Professor Shi-Yin Long or Dr Min Zhang, Department of Biochemistry and Molecular Biology, Medical College, University of South China, 28 West Changsheng Road, Hengyang, Hunan 421001, P.R. China

E-mail: longshiyin@126.com

E-mail: hfpld@163.com

${ }^{*}$ Contributed equally

Abbreviations: ALPs, autolipophagosomes; AS, atherosclerosis; ECs, endothelial cells; HUVECs, human umbilical vein endothelial cells; 7KC, 7-keto cholesterol; LAMP1, lysosomal-associated membrane protein 1; LDs, lipid droplets; ox-LDL, oxidized low-density lipoprotein; TEM, transmission electron microscopy

Key words: oxidized low-density lipoprotein, lipophagy, endothelial dysfunction, oxidative stress injury attenuated and subsequently result in lipid accumulation in ox-LDL-treated ECs.

\section{Introduction}

Lipid accumulation in the arterial walls is one of the hallmarks of atherosclerosis (AS) (1), which is an important pathological manifestation of cardiovascular disease and a notable cause of mortality in numerous countries, such as the UK, Australia and USA (2). It has been demonstrated that monolayer endothelial cell (EC) dysfunction on the inner wall of arterial vessels is the initial step in the process of atherosclerotic plaque formation $(3,4)$. EC dysfunction can be caused by oxidized low-density lipoprotein (ox-LDL), which is often induced by oxidative stress that can be caused by reactive oxygen species, smoking, diabetes and obesity (5-8). Under normal conditions, ECs can export hydrolysed LDL-derived cholesterol into extracellular high-density lipoprotein (HDL), but cannot efficiently efflux ox-LDL-derived cholesterol, resulting in lipid accumulation in ECs (9). Furthermore, the accumulation of ox-LDL in the endothelium has been significantly associated with the dysfunction and apoptosis of ECs $(10,11)$.

Autophagy is a process of evolutionarily conserved catabolism, which degrades a number of cellular components, including long-lived proteins and various organelle components $(12,13)$. A high LC3-II to LC3-I ratio reflects increasing autophagosome formation, whereas low p62 protein levels are associated with high autophagy flux $(14,15)$. LAMP1 is an essential component of the lysosomal membrane, serving as a lysosomal biomarker with a key role in autophagy and lysosomal fusion (16). It has been demonstrated that autophagy can protect ECs from oxidative stress-induced cell damage (17). Since ox-LDL has been reported to be involved in the development of AS and the rupture of atherosclerotic plaques by inhibiting autophagy (18), it was hypothesized that endothelial autophagy serves a key role in limiting the accumulation of ox-LDL-associated lipids within vessel walls (19). An association between ox-LDL-associated autophagy and endothelial lipid accumulation has been identified; however, the mechanism remains unclear.

The specific degradation of lipids by autophagy (also known as lipophagy) was first described in the liver, where 
genetic defects (mutations in RNAi-ATG5 and ATG7) of macro-autophagy led to the accumulation of lipid droplets (LDs) (20). Lipophagy serves a critical role in maintaining overall lipid homeostasis (21). It has been demonstrated that mice bearing autophagy-related 5-deficient macrophages exhibit increased plaque formation due to an impairment of lipophagy $(22,23)$. Therefore, the present study hypothesized that the decrease in endothelial autophagy induced by ox-LDL may be due to impaired lipophagy, resulting in intracellular lipid accumulation and EC damage.

The current study investigated the association between autophagy and lipid accumulation in human umbilical vein endothelial cells (HUVECs) treated with ox-LDL. The results provide evidence that lipophagy may be attenuated in HUVECs with prolonged exposure to ox-LDL and may provide novel insights into the prevention and treatment of atherosclerotic cardiovascular disease.

\section{Materials and methods}

Cell culture and treatments. HUVECs were purchased from The Cell Bank of Type Culture Collection of the Chinese Academy of Sciences and were maintained at $37^{\circ} \mathrm{C}$ with $5 \%$ $\mathrm{CO}_{2}$ in high glucose DMEM supplemented with $10 \%$ FBS (both Gibco; Thermo Fisher Scientific, Inc.), 100 U/ml penicillin and $100 \mu \mathrm{g} / \mathrm{ml}$ streptomycin. HUVECs were treated with PBS (basal), LDL (50 $\mu \mathrm{g} / \mathrm{ml}$; control) or ox-LDL (100 $\mu \mathrm{g} / \mathrm{ml})$ for $6,12,24$ and $48 \mathrm{~h}$.

Cell viability assay. An MTT assay was used to determine the viability of HUVECs. Cells were seeded into 96-well microplates at a density of $4 \times 10^{4}$ cells/well and cultured $37^{\circ} \mathrm{C}$ for $24 \mathrm{~h}$. Subsequently, HUVECs were treated with different concentrations of ox-LDL (25, 50 or $100 \mu \mathrm{g} / \mathrm{ml})$ for 6,12 , 24 and $48 \mathrm{~h}$, and $20 \mu 1$ MTT solution $(5 \mathrm{mg} / \mathrm{ml}$; Beyotime Institute of Biotechnology) was added to each well. The plates were incubated at $37^{\circ} \mathrm{C}$ for $4 \mathrm{~h}$ and then DMSO was added to dissolve the purple formazan products. The optical density value at $490 \mathrm{~nm}$ was measured using a microplate reader spectrophotometer (Molecular Devices, LLC).

Transmission electron microscopy (TEM). After removing the culture medium, the HUVECs were washed twice with ice-cold PBS and fixed with $2.5 \%$ glutaraldehyde overnight at $4^{\circ} \mathrm{C}$. The cells were post-fixed in $2 \%$ osmium tetroxide at $4^{\circ} \mathrm{C}$ for $1 \mathrm{~h}$. Dehydration was subsequently performed in $50-90 \%$ ethanol, and the samples were embedded in epoxy resin at $45^{\circ} \mathrm{C}$ for $12 \mathrm{~h}$. Ultrathin sections (70-nm) were cut using an ultramicrotome (Leica Microsystems $\mathrm{GmbH}$ ) and counterstained with uranyl acetate for $10 \mathrm{~min}$ and lead citrate for $30 \mathrm{~min}$ at RT. The sections were examined using a Jeol JEM SX 100 electron microscope (magnification, x1,200) (JEOL, Ltd.).

Oil Red $O$ staining. The HUVECs were seeded into 6-well plates $\left(2.5 \times 10^{5}\right.$ cells/well) and were treated as aforementioned. The cells were washed twice with PBS and fixed with $4 \%$ paraformaldehyde for $20 \mathrm{~min}$ at room temperature. Subsequently, the HUVECs were stained with a $0.3 \%$ Oil Red $\mathrm{O}$ staining solution for $8 \mathrm{~min}$ at room temperature and washed with $60 \%$ isopropanol for $5 \mathrm{sec}$. The cells were stained with

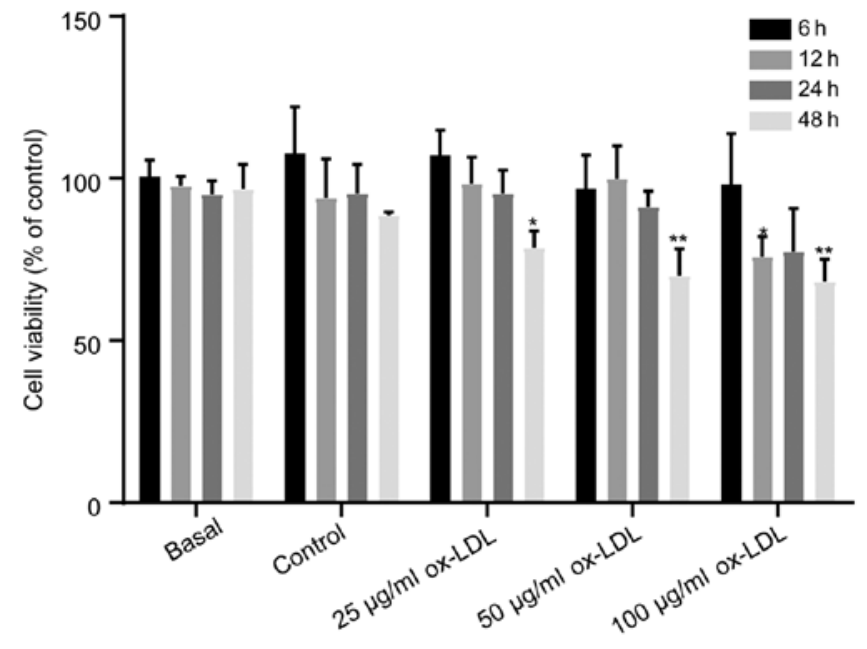

Figure 1. HUVEC viability is decreased by ox-LDL. HUVECs were treated with either PBS (basal group), $50 \mu \mathrm{g} / \mathrm{ml} \mathrm{LDL}$ (control group) or 25, 50 or $100 \mu \mathrm{g} / \mathrm{ml}$ ox-LDL for $6,12,24$ or $48 \mathrm{~h} .{ }^{*} \mathrm{P}<0.05 ;{ }^{* * *} \mathrm{P}<0.01$ vs. control group $(n=3)$. HUVEC, human umbilical vein endothelial cell; ox-LDL, oxidized low-density lipoprotein.

haematoxylin (Sigma-Aldrich; Merck KGaA) for $10 \mathrm{sec}$ at RT prior to obtaining images using a light microscope (magnification, $\mathrm{x} 40$ ) (Nikon Corporation).

Western blot analysis. Total protein was extracted from HUVECs using a lysis buffer $(20 \mathrm{mM}$ Tris- $\mathrm{HCl} \mathrm{pH} \mathrm{7.5,150} \mathrm{mM}$ $\mathrm{NaCl}, 1 \%$ Triton X-100, $1 \mathrm{mM}$ phenylmethylsulphonyl fluoride, $1 \mathrm{mM} \mathrm{Na} \mathrm{VO}_{4}$, leupeptin and EDTA) and was quantified using a bicinchoninic acid kit (Beijing ComWin Biotech Co., Ltd.). Equal amounts of proteins ( $30 \mu \mathrm{g} / \mathrm{lane})$ were separated using $8-12 \%$ SDS-PAGE and transferred onto polyvinylidene difluoride membranes (EMD Millipore). Following blocking with 5\% BSA (Sigma-Aldrich; Merck KGaA) for $1.5 \mathrm{~h}$ at room temperature, the membranes were incubated with the following primary antibodies (all 1:1,000) at $4^{\circ} \mathrm{C}$ overnight: Anti-LC3B (cat. no. 3868S; Cell Signaling Technology, Inc.), anti-p62 (cat. no. ab91526; Abcam), anti-lysosomal-associated membrane protein 1 (LAMP1; cat. no. ab24170; Abcam) and anti- $\beta$-actin (cat. no. 4970S; Cell Signaling Technology, Inc.). Subsequently, the membranes were incubated with an anti-rabbit IgG HRP-linked secondary antibody (1:5,000; cat. no. 7074; Cell Signalling Technology) for $45 \mathrm{~min}$ at room temperature. The bands were visualized using an enhanced chemiluminescence reagent (EMD Millipore). The densitometry of each band was analysed using the Sigma Scan Pro5 software (version 5.0; Systat Software, Inc.) and normalized to $\beta$-actin values.

Immunofluorescence staining. The HUVECs were fixed with $4 \%$ paraformaldehyde ( $\mathrm{pH}$ 7.4) for $20 \mathrm{~min}$ at RT, washed three times with PBS, blocked with 5\% BSA at RT for $2 \mathrm{~h}$, and then incubated with the following rabbit primary antibodies (all 1:200) overnight at $4^{\circ} \mathrm{C}$ : Anti-LAMP 1 (cat. no. ab24170; Abcam) and anti-LC3B (cat. no. 3868S; Cell Signaling Technology, Inc.). Subsequently, the cells were incubated with anti-rabbit $\operatorname{IgG}(\mathrm{H}+\mathrm{L}), \mathrm{F}\left(\mathrm{ab} \mathrm{b}^{\prime}\right) 2$ Fragment (Alexa Fluor ${ }^{\circledR}$ 647 Conjugate) (red) antibodies (1:500; cat. no. 4414; Cell 

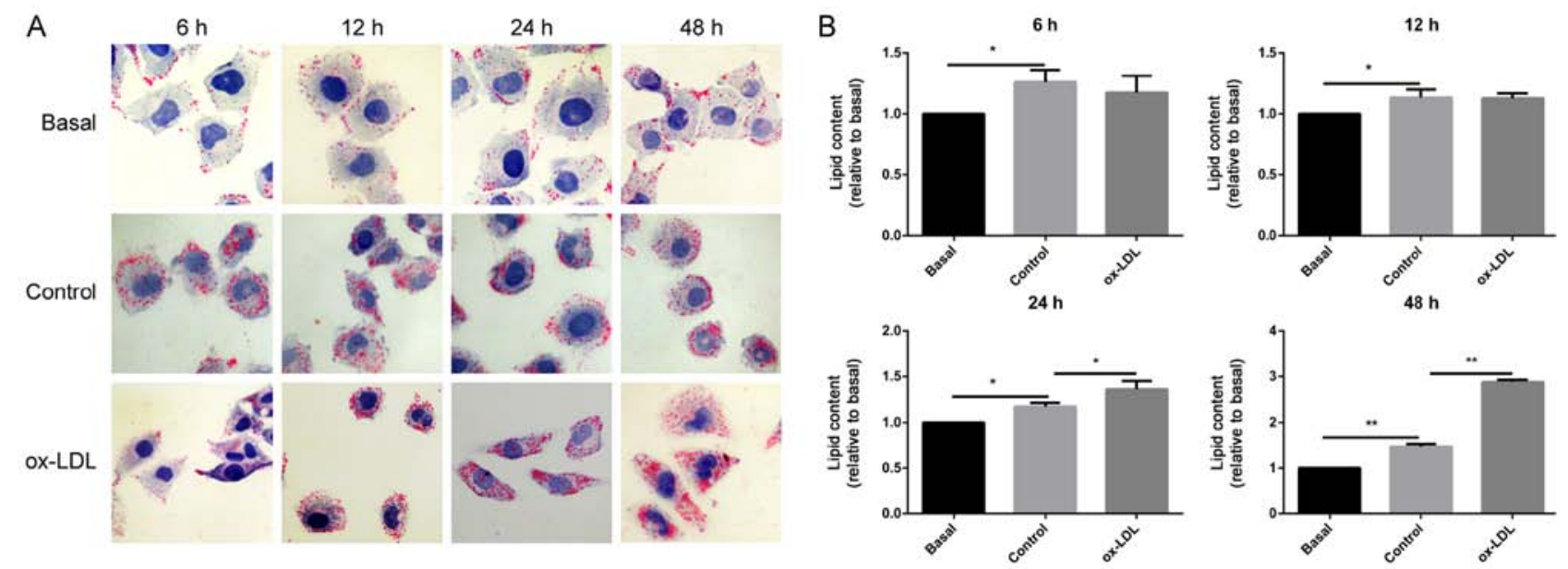

Figure 2. Neutral lipid accumulation of the HUVECs due to ox-LDL. HUVECs were treated with either PBS (basal group), $50 \mu \mathrm{g} / \mathrm{ml}$ LDL (control group) or $100 \mu \mathrm{g} / \mathrm{ml}$ ox-LDL for 6,12, 24 or $48 \mathrm{~h}$. (A) Lipid accumulation was detected using Oil Red O staining. (B) Quantification of neutral lipids was analysed using Fiji software. Magnification, $\mathrm{x} 40 .{ }^{~} \mathrm{P}<0.05 ;{ }^{* *} \mathrm{P}<0.01(\mathrm{n}=3)$. HUVEC, human umbilical vein endothelial cell; ox-LDL, oxidized low-density lipoprotein.

Signaling Technology) for $1 \mathrm{~h}$ at $37^{\circ} \mathrm{C}$. LDs were stained with $1 \mu \mathrm{g} / \mathrm{ml}$ Bodipy 493/503 (Invitrogen; Thermo Fisher Scientific, Inc.) for $30 \mathrm{~min}$ at RT in the dark, and with $10 \mathrm{mg} / \mathrm{ml}$ DAPI (cat. no. H-1200; Vector Laboratories, Inc.) at RT for $5 \mathrm{~min}$. The cells were examined under a confocal laser scanning microscope (magnification, x400) (LSCM 510 Meta; Zeiss AG). The relative colocalization coefficient between LC3, LAMP1 and LD was analysed via ImageJ (version no. 1.52P) software (24).

Statistical analysis. All values were presented as the mean $\pm S D(n \geq 3)$. The differences between groups were analysed via one-way ANOVA and post hoc Dunnett's multiple comparisons test by GraphPad Prism (version 6; GraphPad Software, Inc.). $\mathrm{P}<0.05$ was considered to indicate a statistically significant difference.

\section{Results}

Lipid accumulation in HUVECs induced by ox-LDL. To determine the appropriate concentration of ox-LDL, as an oxidative stress factor on ECs, the HUVECs were treated with different concentrations of ox-LDL $(25,50$ or $100 \mu \mathrm{g} / \mathrm{ml})$ for $6,12,24$ and $48 \mathrm{~h}$, and the cell viability was detected using the MTT assay. The results demonstrated that there was no difference in cell viability in HUVECs treated with $25 \mu \mathrm{g} / \mathrm{ml}$ ox-LDL for 6,12 , and $24 \mathrm{~h}$, whereas compared with corresponding control group, $50 \mu \mathrm{g} / \mathrm{ml}$ ox-LDL for $48 \mathrm{~h}$ inhibited cell growth (Fig. 1). Furthermore, when treated with $100 \mu \mathrm{g} / \mathrm{ml}$ ox-LDL, the cell viability significantly decreased at 12 and $48 \mathrm{~h}$ compared with the control group (Fig. 1), which is consistent with the finding that $100 \mu \mathrm{g} / \mathrm{ml}$ ox-LDL decreased HUVEC viability when investigating the effect of ox-LDL on autophagy (25). Therefore, $100 \mu \mathrm{g} / \mathrm{ml}$ ox-LDL was chosen as the oxidative stress factor for all subsequent experiments.

To investigate the deposited lipid effect on the ECs under oxidative stress, the LDs were stained with Oil Red O in HUVECs that had been exposed to $100 \mu \mathrm{g} / \mathrm{ml}$ ox-LDL. As shown in Fig. 2, the results demonstrated that compared with the basal group, lipid droplets in HUVECs treated with LDL for
$6,12,24$ and $48 \mathrm{~h}$ were significantly increased, which indicates that HUVECs were lipid-loaded. Lipid staining in HUVECs showed a decreased tendency without statistical difference following treatment with ox-LDL for $6 \mathrm{~h}$ and $12 \mathrm{~h}$ compared with that in cells treated with LDL (control). However, when the HUVECs were treated with ox-LDL for 24 and $48 \mathrm{~h}$, lipid deposition in the cells was significantly increased compared with that in the control group. The present data indicated that prolonged exposure to ox-LDL led to elevated lipid accumulation in the ECs.

Impaired autophagy in HUVECs due to ox-LDL. To investigate the effect of ox-LDL on autophagy in HUVECs, the ratio of LC3-II to LC3-I and the p62 protein expression levels, which serve as well-known biomarkers of autophagy (26), were detected using western blot analysis. As shown in Fig. 3, the results demonstrated that compared with LDL exposure, the ratio of LC3II/LC3I was significantly upregulated in HUVECs exposed to ox-LDL for $6 \mathrm{~h}$, along with significantly increased protein expression levels of LAMP1 and decreased protein levels of p62, suggesting that autophagy events may be triggered in the early period of oxidative stress exposure in ECs. However, compared with the control group, decreased protein levels of LAMP1 were observed in HUVECs exposed to ox-LDL for $24 \mathrm{~h}$, which was accompanied by increased $\mathrm{p} 62$ expression. Furthermore, there was a significant decrease in both the ratio of LC3II/LC3I and the protein expression levels of LAMP1, along with a significant increase in p62 expression, in HUVECs treated with ox-LDL for $48 \mathrm{~h}$, indicating that autophagy may be inhibited with prolonged exposure of ECs to ox-LDL.

Suppression of lipophagy in HUVECs treated with ox-LDL. To clarify the association between ox-LDL-induced autophagy deficiency and lipid accumulation in ECs, the changes in the number of autolipophagosomes (ALPs) were detected using TEM. The results in Fig. 4 revealed that ALP formation in the ox-LDL treatment group was higher compared with that in the LDL treatment group following $6 \mathrm{~h}$ of treatment. However, this ultra-structural conformation was decreased with exposure to 


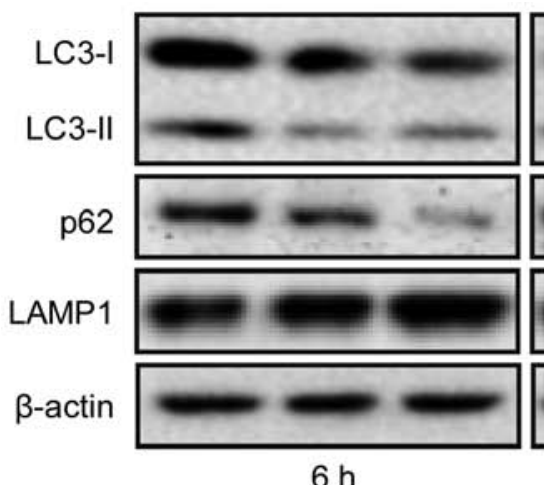

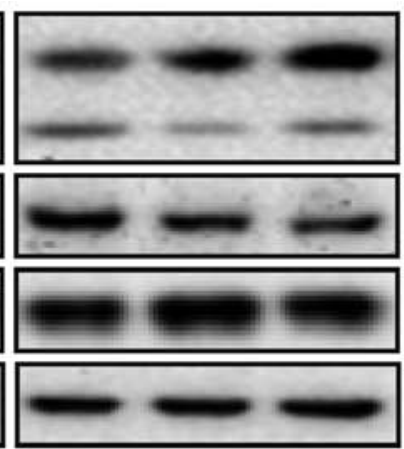

$12 \mathrm{~h}$

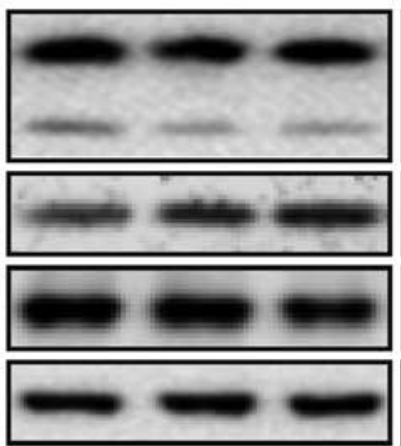

$24 \mathrm{~h}$

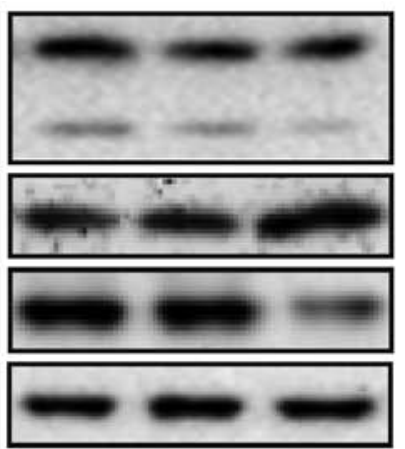

$48 \mathrm{~h}$
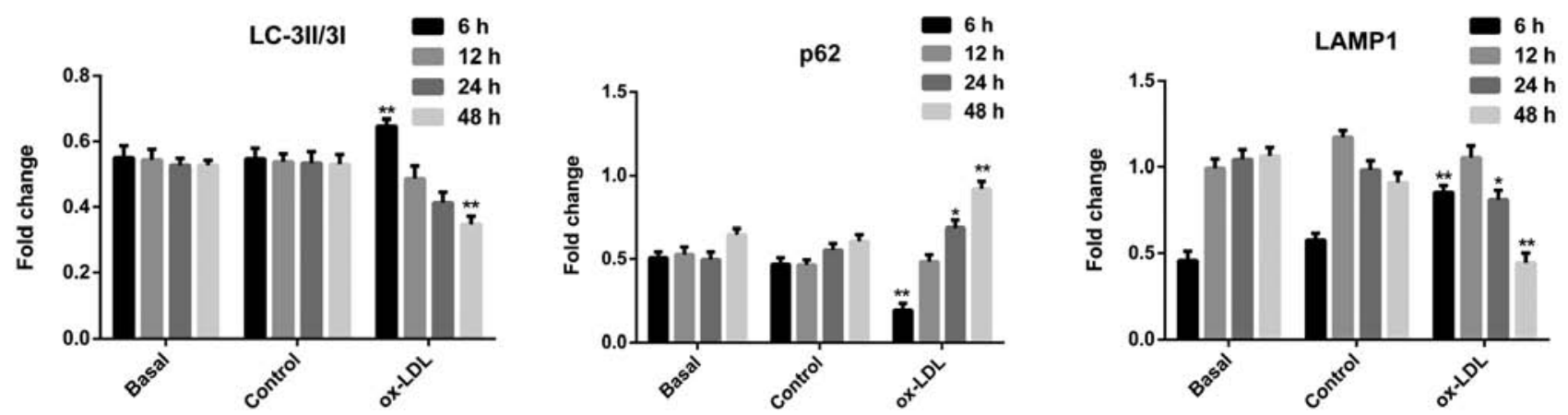

Figure 3. Effect of ox-LDL on the expression of LC3-II/LC3-I, p62 and LAMP1 in HUVECs. HUVECs were treated with either PBS (basal group), 50 $\mu \mathrm{g} / \mathrm{ml}$ LDL (control group) or $100 \mu \mathrm{g} / \mathrm{ml}$ ox-LDL for $6,12,24$ or $48 \mathrm{~h}$. Expression levels of autophagy-associated proteins (LC3II/I and p62) and LAMP1 were analysed using western blot analysis. The ratio of LC3-II/LC3-I, p62 protein expression and LAMP1 protein expression were quantified using the Fiji software. ${ }^{*} \mathrm{P}<0.05 ;{ }^{* *} \mathrm{P}<0.01$ vs. control group ( $\left.\mathrm{n}=3\right)$. HUVEC, endothelial cell; ox-LDL, oxidized low-density lipoprotein; LAMP1, lysosomal-associated membrane protein 1.

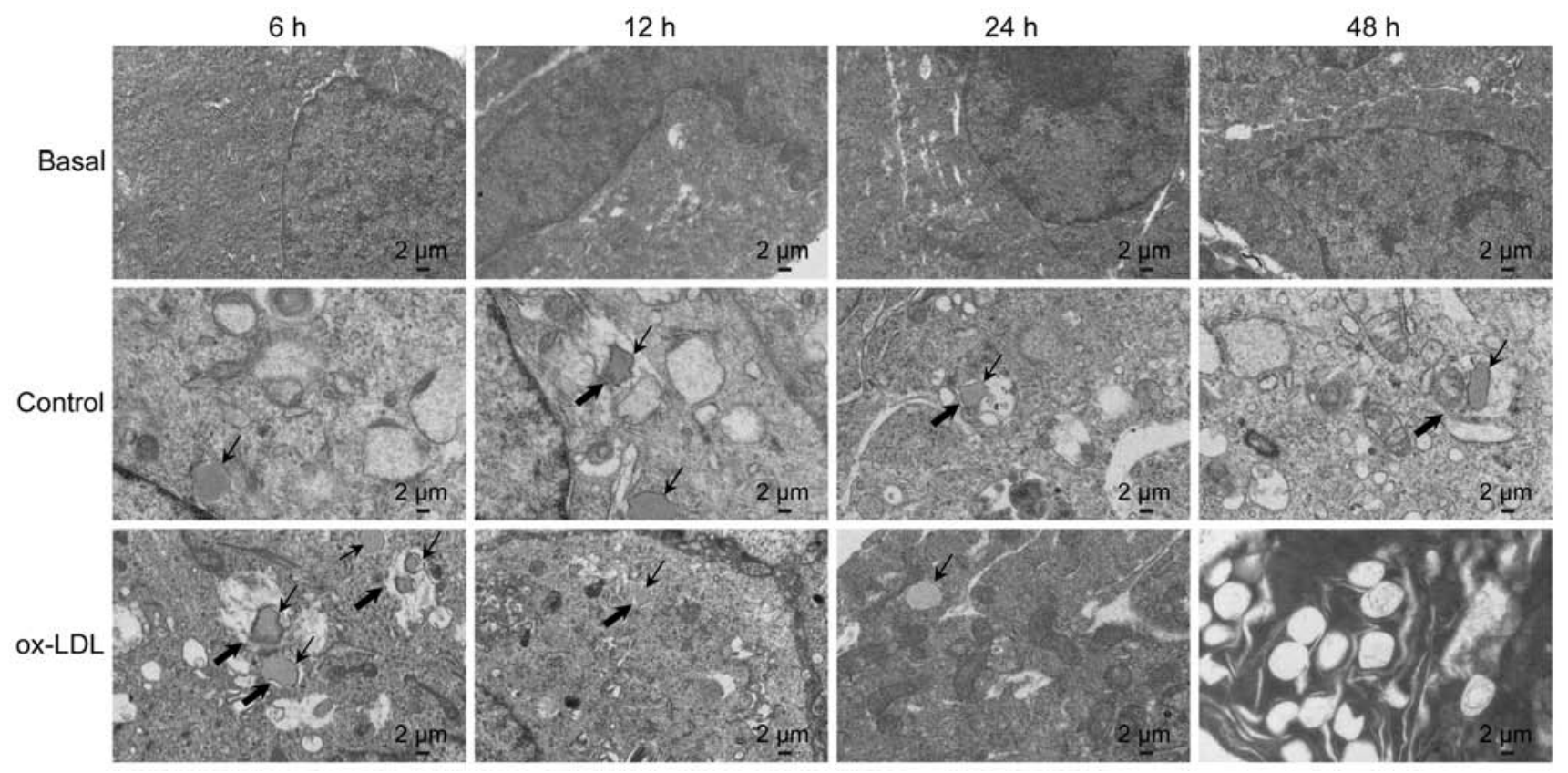

Figure 4. Reduction of lipophagy in HUVECs when exposed to ox-LDL. HUVECs were untreated (basal group) or treated with either $50 \mu \mathrm{g} / \mathrm{ml} \mathrm{LDL}$ (control group) or $100 \mu \mathrm{g} / \mathrm{ml}$ ox-LDL for 6, 12, $24 \mathrm{or} 48 \mathrm{~h}$. Scale bar, $2 \mu \mathrm{m}$. Thick arrows indicate autolipophagosome formation, and thin arrows indicate lipid droplets ( $n=3$ per group). HUVEC, endothelial cell; ox-LDL, oxidized low-density lipoprotein.

ox-LDL for 24 and $48 \mathrm{~h}$ compared with that in LDL-treated HUVECs. In addition, following exposure to ox-LDL for $48 \mathrm{~h}$, the signs of damage was observed in ECs by the presence of vacuole-like structures. The present results suggested that lipophagy may be impaired after prolonged exposure to oxidative stress. 

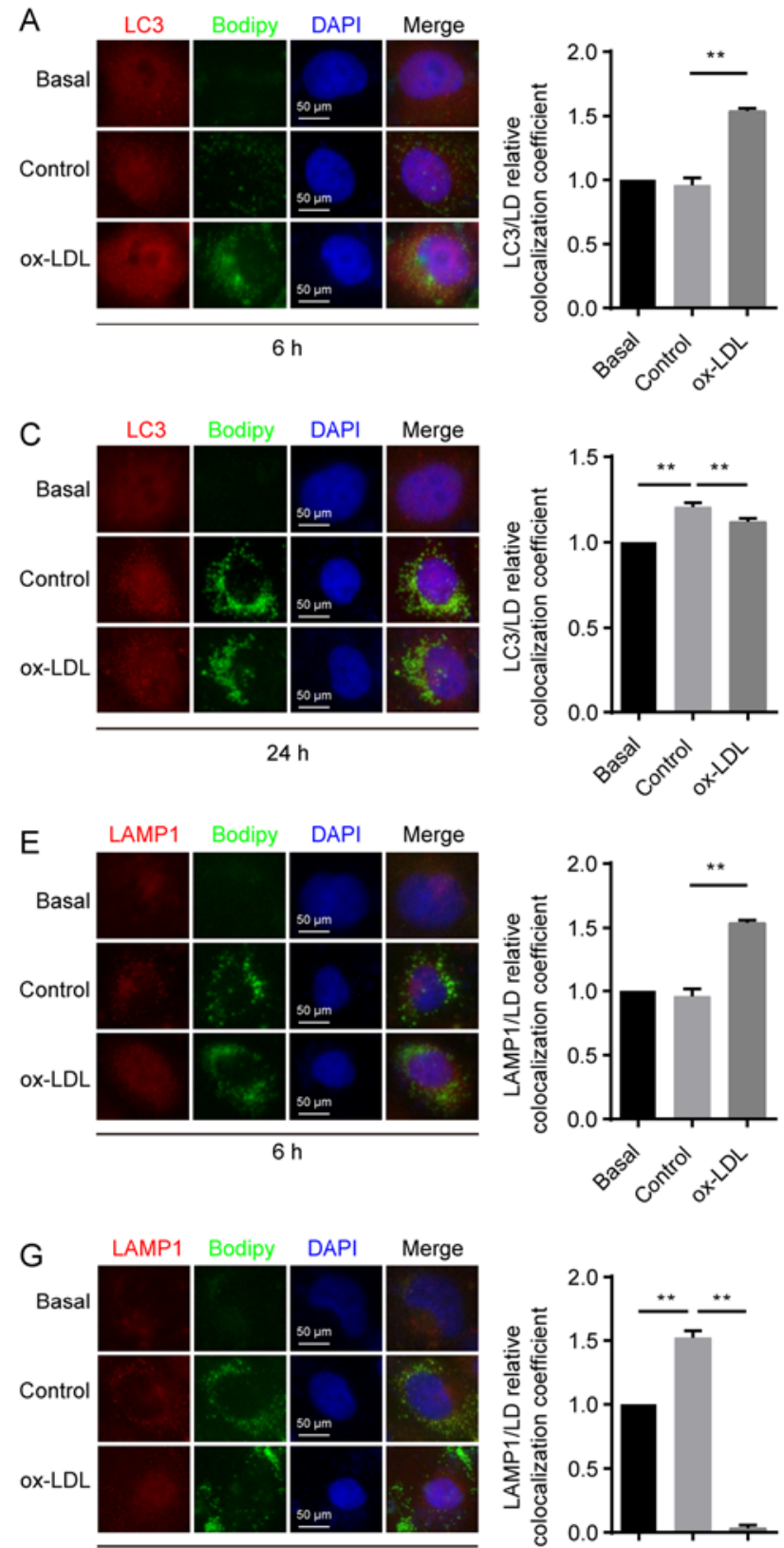

$24 \mathrm{~h}$
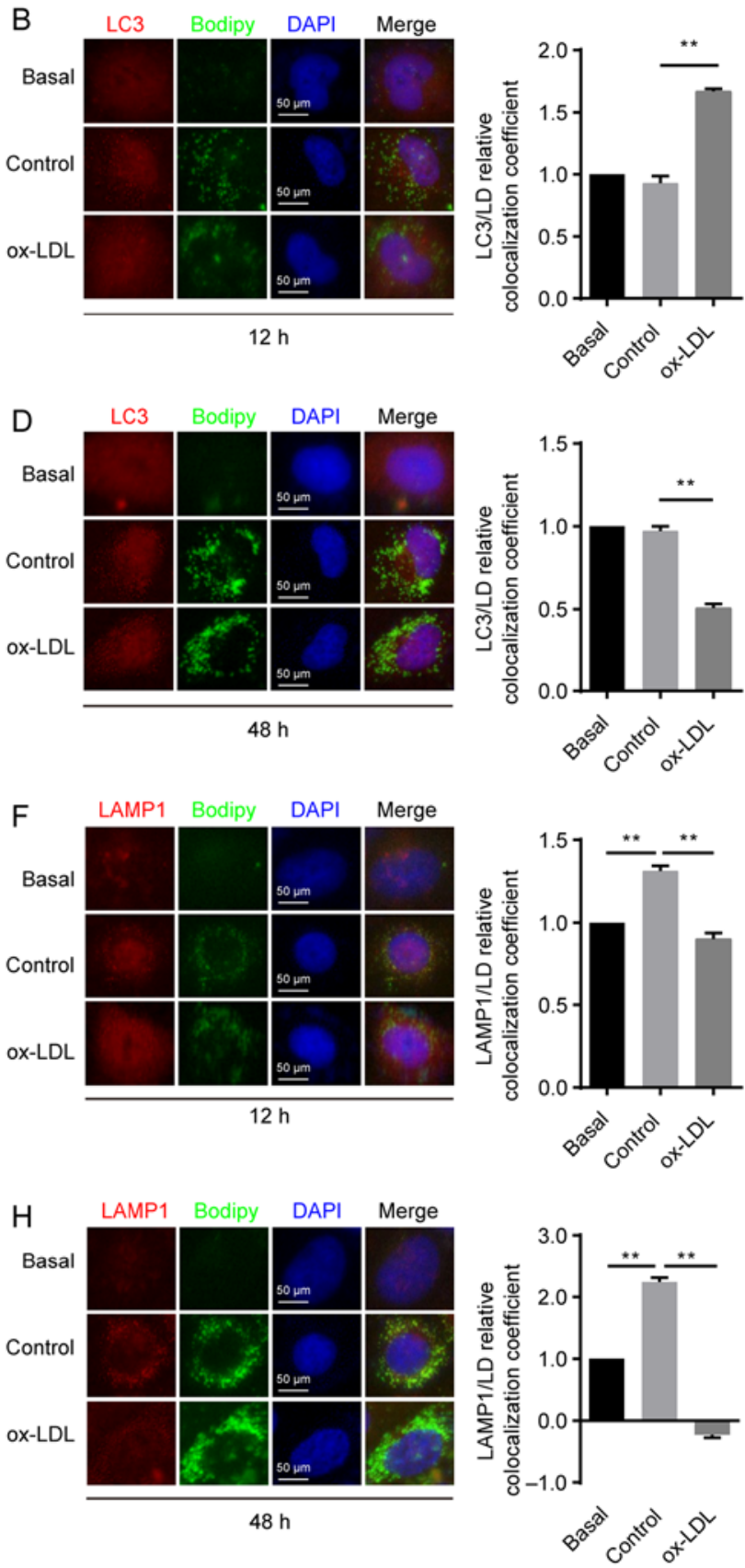

Figure 5. LD delivery to lysosomes for degradation is impaired in HUVECs exposed to ox-LDL. HUVECs were untreated (basal) or treated with either $50 \mu \mathrm{g} / \mathrm{ml} \mathrm{LDL} \mathrm{(control} \mathrm{group)} \mathrm{or} 100 \mu \mathrm{g} / \mathrm{ml}$ ox-LDL for 6, 12, 24 or $48 \mathrm{~h}$. Co-localization of LC3 (red) and LDs (green) in HUVECs following exposure to ox-LDL for (A) 6, (B) 12, (C) 24 and (D) 48 h. Co-localization of LAMP1 (red) and LDs (green) in HUVECs following exposure to ox-LDL for (E) 6, (F) 12, (G) 24 and (H) 48 h. Magnification, $x 400 .{ }^{* *} \mathrm{P}<0.01(\mathrm{n}=3)$. HUVEC, endothelial cell; ox-LDL, oxidized low-density lipoprotein; LD, lipid droplet; LAMP1, lysosomal-associated membrane protein 1.

Impaired lipophagymay be associated with lipidaccumulation in ECs. To further elucidate the localization of LDs in HUVECs treated with ox-LDL, the co-localization of LDs with LC3 or LAMP1 was measured using confocal microscopy, as previously described (27). Compared with the basal group, the co-localization of LDs with LC3 was significantly increased in HUVECs treated with LDL for $24 \mathrm{~h}$ (Fig. 5C). In addition, this co-localization was increased with exposure to ox-LDL for 6 and $12 \mathrm{~h}$ compared with that in the control group, (Fig. 5A and B); however, decreased co-localization of LDs with LC3 was observed with exposure to ox-LDL for 24 and $48 \mathrm{~h}$ (Fig. 5C and D), indicating that oxidative stress may reduce lipophagy in HUVECs when treated with ox-LDL for a longer time.

As the exposure time of HUVECs to LDL was increased, the co-localization of LDs with LAMP1 was significantly increased at both 12 and $24 \mathrm{~h}$ compared with that in the basal group (Fig. 5E-H), which demonstrated that treatment with natural LDL may accelerate the process of lipid degradation in the lysosomes. Compared with the control group, the co-localization of LDs with LAMP1 was significantly increased in ECs treated with ox-LDL for $6 \mathrm{~h}$ (Fig. 5E); however, this co-localization was significantly decreased when increasing the exposure time from 12 to $48 \mathrm{~h}$ (Fig. 5F, G and $\mathrm{H}$ ). The 
present results demonstrated that ox-LDL induced by oxidative stress may hinder lipid degradation in the ECs lysosomes.

Overall, the increased lipophagy resulting from exposure to ox-LDL for 6 to $12 \mathrm{~h}$ may be associated with enhanced degradation of ox-LDL via autophagy-lysosomal pathways, and the decreased lipophagy observed with exposure to ox-LDL for 24 and $48 \mathrm{~h}$ may be associated with attenuated degradation of ox-LDL via autophagy-lysosomal pathways. This phenomenon can be explained by the fact that the lysosomal pathway is associated with decreased ox-LDL degradation, while lipids are degraded in autophagy-lysosomal pathways (28).

\section{Discussion}

AS is a major cause of cardiovascular diseases and typically leads to stroke, myocardial infarction and coronary heart disease (2). Damaged ECs lose the barrier function of the arterial endothelium, accelerating the development of AS (29). Furthermore, ECs are highly sensitive to ox-LDL-induced oxidative damage (30), which is associated with the risk of coronary heart disease and myocardial infarction (31). In addition to being an oxidative stress factor, ox-LDL particles are recognized and captured by scavenger receptors on ECs, rather than by LDL receptors, via a reverse cholesterol transport mechanism (32). It has been previously demonstrated that ox-LDL promotes apoptosis, which disrupts the integrity of the arterial endothelium (33).

The present study demonstrated that exposure to a high concentration of ox-LDL $(100 \mu \mathrm{g} / \mathrm{ml})$ decreased the viability of HUVECs and increased lipid deposition, whereas natural LDL had no effect on the activity of HUVECs. The present results suggested that oxidative stress may damage ECs, further leading to lipid accumulation. It has been established that 7-keto cholesterol $(7 \mathrm{KC})$, one of the components of ox-LDL which has a similar effect on ECs to total ox-LDL (34), is also found in human atherosclerotic plaques and serves an active role in plaque development via inducing vascular EC apoptosis (35). Therefore, it is important to develop strategies to reduce the lipid accumulation induced by ox-LDL.

Both autophagy and apoptosis are associated with maintaining normal cell functions (36). Autophagy inhibits apoptosis to ensure cell survival and it is also an alternative mechanism for cell death in the absence of cell apoptosis (37). In addition, autophagy can protect various vascular cells, such as macrophages and vascular smooth muscle cells, against oxidative stress and inflammation, slowing down atherosclerotic processes (38). It has been demonstrated that autophagy can be induced by $7 \mathrm{KC}$ to inhibit the death of smooth muscle cells and to avoid thinning of the fibrous cap (39). Furthermore, it has been revealed that ox-LDL inhibits the autophagy of ECs, serving an essential role in the development of atherosclerotic lesions (12). However, the precise mechanism by which ox-LDL inhibits autophagy in ECs has not yet been elucidated. In the present study, the potential role of autophagy in regulating ox-LDL-induced ECs was investigated. It was revealed that when treating HUVECs with ox-LDL for 24 and $48 \mathrm{~h}$, the ratio of LC3-II/I protein expression levels were significantly decreased, but the protein expression levels of p62 were significantly increased, indicating that autophagy was significantly impaired. After autophagy is induced, autophagosomes are fused with lysosomes to form autolysosomes, in which the cargo of the autophagosomes is ultimately degraded (40). LAMP1 is an essential factor that mediates the fusion between autophagosomes and lysosomes (16). Treatment with ox-LDL for 24 and $48 \mathrm{~h}$ revealed that LAMP1 expression was reduced in HUVECs, which is consistent with previous research (25). Conversely, previous studies demonstrate that autophagy induced by rapamycin can decrease ox-LDL accumulation in ECs (41-43). Overall, the present data indicate that autophagy may be inhibited by ox-LDL in ECs.

Previous studies have revealed that autophagy and lipid accumulation serve important roles in the development of atherosclerotic vascular diseases $(44,45)$. Of note, lipid accumulation has been revealed to accelerate AS development (45); however, the exact mechanism of this process remains unclear. In the present study, the underlying role of autophagy in regulating ox-LDL-induced EC lipid accumulation was explored, and the intermolecular interactions were investigated. Increased lipid staining was observed around the nucleus of HUVECs treated with ox-LDL for 24 and 48 h, suggesting that there may be an association between autophagy and lipid accumulation in ECs exposed to ox-LDL.

Lipophagy is a special form of autophagy (46), which may be involved in transporting LDs from cells to extracellular HDL. To investigate whether lipophagy was impaired under ox-LDL prolonged exposure, numerous ultrastructural changes in ECs were observed using TEM. Compared with control cells, fewer ALPs were observed in cells following treatment with ox-LDL for 24 and $48 \mathrm{~h}$, after which they also exhibited features of cell damage, such as an excess in the number of vacuoles. The present results suggested that lipophagy may be attenuated by ox-LDL, which subsequently promoted lipid accumulation in ECs.

To confirm the impaired lipophagy, the co-localization of LC3, LDs and LAMP1 were assessed using immunofluorescence staining in HUVECs. Compared with the basal group, the co-localization of LDs with LC3 was not significantly different in HUVECs treated with LDL for 6, 12, and $48 \mathrm{~h}$, which suggested that lipophagy occurs under normal LDL exposure. Whereas following treatment with ox-LDL for 24 and $48 \mathrm{~h}$, the co-localization of LC3 and LDs was significantly decreased, as well as the co-localization of LAMP1 and LDs. The present result suggested that endothelial lipophagy may partially maintain vascular lipid homeostasis on autophagosomal-mediated delivery of lipids to the lysosome for degradation (19). Furthermore, lipophagy accelerates lipid hydrolysis, which indirectly promotes the release of free cholesterol to extracellular Apolipoprotein (Apo) AI receptors $(47,48)$.

In conclusion, compared with the control group, lipophagy was impaired in HUVECs treated with ox-LDL, resulting in lipid accumulation. It was hypothesized that lipophagy may be attenuated by ox-LDL, resulting in excess cholesterol deposition in ECs, which may be attributed to inhibiting cholesterol efflux to Apo AI receptors. Overall, the regulation of lipophagy may be an attractive therapeutic strategy to limit atherosclerotic disease in the future. 


\section{Acknowledgements}

The authors would like to thank DrZhi-xin Huang (Guangdong Second Provincial General Hospital, Guangzhou, China) for assisting with statistical analysis; Dr Hong An (University of Missouri, Columbia, MO, USA) for helping to modify the manuscript; Dr Tan Tan and Dr Xiao-bo Hu (University of South China, Hengyang, China) for technical support.

\section{Funding}

The present study was supported by the National Nature Science Fund of China (grant nos. 81600291 and 31871169), the Nature Science Fund of Hunan province (grant nos. 2018JJ2346 and 2018JJ2348) and the Hunan Provincial Innovation Foundation for Postgraduate (grant no. CX2018B62).

\section{Availability of data and materials}

The datasets used and/or analyzed during the current study are available from the corresponding author on reasonable request.

\section{Authors' contributions}

SYL, MZ and CPZ conceptualized and designed the experiments. XXD, TT and CPZ performed the experiments. BJL, CYW and SSJ analyzed and interpreted the data. JQS and YLY aquired the data. CPZ and XXD wrote the manuscript. YT made substantial contributions to conception of the study and critically revised the manuscript for important intellectual content. All authors read and approved the final version of the manuscript.

\section{Ethics approval and consent to participate}

Not applicable.

\section{Patient consent for publication}

Not applicable.

\section{Competing interests}

The authors declare that they have no competing interests.

\section{References}

1. Libby P, Ridker PM and Hansson GK: Progress and challenges in translating the biology of atherosclerosis. Nature 473 : 317-325, 2011

2. Reamy BV, Williams PM and Kuckel DP: Prevention of cardiovascular disease. Prim Care 45: 25-44, 2018.

3. Yamagishi SI and Matsui T: Anti-atherothrombogenic properties of PEDF. Curr Mol Med 10: 284-291, 2010.

4. Tsaousi A, Mill C and George SJ: The Wnt pathways in vascular disease: Lessons from vascular development. Curr Opin Lipidol 22: 350-357, 2011.

5. Ding L, Biswas S, Morton RE, Smith JD, Hay N, Byzova TV, Febbraio M and Podrez EA: Akt3 deficiency in macrophages promotes foam cell formation and atherosclerosis in mice. Cell Metab 15: 861-872, 2012.
6. Williams HJ, Fisher EA and Greaves DR: Macrophage differentiation and function in atherosclerosis: Opportunities for therapeutic intervention? J Innate Immun 4: 498-508, 2012.

7. Hellings WE, Peeters W, Moll FL, Piers SR, van Setten J, Van der Spek PJ, de Vries JP, Seldenrijk KA, De Bruin PC, Vink A, et al: Composition of carotid atherosclerotic plaque is associated with cardiovascular outcome: A prognostic study. Circulation 121: 1941-1950, 2010.

8. Mimura $\mathrm{J}$ and Itoh $\mathrm{K}$ : Role of $\mathrm{Nrf} 2$ in the pathogenesis of atherosclerosis. Free Radic Biol Med 88: 221-232, 2015.

9. Voloshyna I, Hai O, Littlefield MJ, Carsons S and Reiss AB: Resveratrol mediates anti-atherogenic effects on cholesterol flux in human macrophages and endothelium via PPARg and adenosine. Eur J Pharmacol 698: 299-309, 2013.

10. Wu CY, Zhou ZF, Wang B, Ke ZP, Ge ZC and Zhang XJ: MicroRNA-328 ameliorates oxidized low-density lipoprotein-induced endothelial cells injury through targeting HMGB1 in atherosclerosis. J Cell Biochem: Oct 15, 2018 (Epub ahead of print).

11. Demers A, Samami S, Lauzier B, Des Rosiers C, Ngo Sock ET, Ong $\mathrm{H}$ and Mayer G: PCSK9 induces CD36 degradation and affects long-chain fatty acid uptake and triglyceride metabolism in adipocytes and in mouse liver. Arterioscler Thromb Vasc Biol 35: 2517-2525, 2015.

12. Che J, Liang B, Zhang Y, Wang Y, Tang J and Shi G: Kaempferol alleviates ox-LDL-induced apoptosis by up-regulation of autophagy via inhibiting PI3K/Akt/mTOR pathway in human endothelial cells. Cardiovasc Pathol 31: 57-62, 2017.

13. Boya P, Reggiori F and Codogno P: Emerging regulation and functions of autophagy. Nat Cell Biol 15: 713-720, 2013.

14. Li X, Li Y, Zhao J, Li L, Wang Y, Zhang Y, Li Y, Chen Y, Liu W and Gao L: Administration of ketamine causes autophagy and apoptosis in the rat fetal hippocampus and in PC12 cells. Front Cell Neurosci 12: 21, 2018.

15. Wang L, Kim D, Wise JTF, Shi X, Zhang Z and DiPaola RS: p62 as a therapeutic target for inhibition of autophagy in prostate cancer. Prostate 78: 390-400, 2018.

16. Fortunato F, Burgers H, Bergmann F, Rieger P, Buchler MW, Kroemer $\mathrm{G}$ and Werner J: Impaired autolysosome formation correlates with Lamp-2 depletion: Role of apoptosis, autophagy, and necrosis in pancreatitis. Gastroenterology 137: 350-360.el-5, 2009.

17. Nowicki M, Zabirnyk O, Duerrschmidt N, Borlak J and Spanel-Borowski K: No upregulation of lectin-like oxidized low-density lipoprotein receptor-1 in serum-deprived EA.hy926 endothelial cells under oxLDL exposure, but increase in autophagy. Eur J Cell Biol 86: 605-616, 2007.

18. Mollace V, Gliozzi M, Musolino V, Carresi C, Muscoli S, Mollace R, Tavernese A, Gratteri S, Palma E, Morabito C, et al: Oxidized LDL attenuates protective autophagy and induces apoptotic cell death of endothelial cells: Role of oxidative stress and LOX-1 receptor expression. Int J Cardiol 184: 152-158, 2015.

19. Torisu K, Singh KK, Torisu T, Lovren F, Liu J, Pan Y, Quan A, Ramadan A, Al-Omran M, Pankova N, et al: Intact endothelial autophagy is required to maintain vascular lipid homeostasis. Aging Cell 15: 187-191, 2016.

20. Singh R: Autophagy and regulation of lipid metabolism. Results Probl Cell Differ 52: 35-46, 2010.

21. Jarc E and Petan T: Lipid droplets and the management of cellular stress. Yale J Biol Med 92: 435-452, 2019.

22. Liao X, Sluimer JC, Wang Y, Subramanian M, Brown K, Pattison JS, Robbins J, Martinez J and Tabas I: Macrophage autophagy plays a protective role in advanced atherosclerosis. Cell Metab 15: 545-553, 2012.

23. Sergin I and Razani B: Self-eating in the plaque: What macrophage autophagy reveals about atherosclerosis. Trends Endocrinol Metab 25: 225-234, 2014.

24. Schindelin J, Arganda-Carreras I, Frise E, Kaynig V, Longair M, Pietzsch T, Preibisch S, Rueden C, Saalfeld S, Schmid B, et al: Fiji: An open-source platform for biological-image analysis. Nat Methods 9: 676-682, 2012.

25. Zhang YL, Cao YJ, Zhang X, Liu HH, Tong T, Xiao GD, Yang YP and Liu CF: The autophagy-lysosome pathway: A novel mechanism involved in the processing of oxidized LDL in human vascular endothelial cells. Biochem Biophys Res Commun 394: 377-382, 2010.

26. Gómez-Sánchez R, Yakhine-Diop SM, Rodríguez-Arribas M, Bravo-San Pedro JM, Martínez-Chacón G, Uribe-Carretero E, Pinheiro de Castro DC, Pizarro-Estrella E, Fuentes JM and González-Polo RA: mRNA and protein dataset of autophagy markers (LC3 and p62) in several cell lines. Data Brief 7: 641-647, 2016. 
27. Singh R and Cuervo AM: Autophagy in the cellular energetic balance. Cell Metab 13: 495-504, 2011.

28. Horie S, Hiraishi S, Hirata Y, Kazama M and Matsuda J: Oxidized low-density lipoprotein impairs the anti-coagulant function of tissue-factor-pathway inhibitor through oxidative modification by its high association and accelerated degradation in cultured human endothelial cells. Biochem J 352: 277-285, 2000.

29. Maracle CX, Agca R, Helder B, Meeuwsen JAL, Niessen HWM Biessen EAL, de Winther MPJ, de Jager SCA, Nurmohamed MT and Tas SW: Noncanonical NF- $\kappa B$ signaling in microvessels of atherosclerotic lesions is associated with inflammation, atheromatous plaque morphology and myocardial infarction. Atherosclerosis 270: 33-41, 2018.

30. Chen B, Meng L, Shen T, Gong H, Qi R, Zhao Y, Sun J, Bao L and Zhao G: Thioredoxin attenuates oxidized low-density lipoprotein induced oxidative stress in human umbilical vein endothelial cells by reducing NADPH oxidase activity. Biochem Biophys Res Commun 490: 1326-1333, 2017.

31. Mitra S, Goyal T and Mehta JL: Oxidized LDL, LOX-1 and atherosclerosis. Cardiovasc Drugs Ther 25: 419-429, 2011.

32. Matsuura E, Hughes GR and Khamashta MA: Oxidation of LDL and its clinical implication. Autoimmun Rev 7: 558-566, 2008.

33. Suc I, Escargueil-Blanc I, Troly M, Salvayre R and Nègre-Salvayre A: HDL and ApoA prevent cell death of endothelial cells induced by oxidized LDL. Arterioscler Thromb Vasc Biol 17: 2158-2166, 1997.

34. Brown AJ and Jessup W: Oxysterols and atherosclerosis Atherosclerosis 142: 1-28, 1999.

35. Sasaki H, Watanabe F, Murano T, Miyashita Y and Shirai K Vascular smooth muscle cell apoptosis induced by 7-ketocholesterol was mediated via $\mathrm{Ca}^{2+}$ and inhibited by the calcium channel blocker nifedipine. Metabolism 56: 357-362, 2007.

36. Ali ES, Rychkov GY and Barritt GJ: Metabolic disorders and cancer: Hepatocyte store-operated $\mathrm{Ca}^{2+}$ channels in nonalcoholic fatty liver disease. Adv Exp Med Biol 993: 595-621, 2017.

37. Wei DH, Jia XY, Liu YH, Guo FX, Tang ZH, Li XH, Wang Z, Liu LS, Wang GX, Jian ZS and Ruan CG: Cathepsin L stimulates autophagy and inhibits apoptosis of ox-LDL-induced endothelial cells: Potential role in atherosclerosis. Int J Mol Med 31: 400-406, 2013

38. Miyazaki T and Miyazaki A: Defective protein catabolism in atherosclerotic vascular inflammation. Front Cardiovasc Med 4: 79, 2017.
39. He C, Zhu H, Zhang W, Okon I, Wang Q, Li H, Le YZ and Xie Z 7-Ketocholesterol induces autophagy in vascular smooth muscle cells through Nox4 and Atg4B. Am J Pathol 183: 626-637, 2013.

40. Gatica D, Lahiri V and Klionsky DJ: Cargo recognition and degradation by selective autophagy. Nat Cell Biol 20: 233-242, 2018.

41. Zhang Y, Han Q, You S, Cao Y, Zhang X, Liu H, Hu L and Liu CF: Rapamycin promotes the autophagic degradation of oxidized low-density lipoprotein in human umbilical vein endothelial cells. J Vasc Res 52: 210-219, 2015.

42. Zhou YD, Cao XQ, Liu ZH, Cao YJ, Liu CF, Zhang YL and Xie Y: Rapamycin inhibits oxidized low density lipoprotein uptake in human umbilical vein endothelial cells via mTOR/NF-кB/LOX-1 pathway. PLoS One 11: e0146777, 2016.

43. Sun JJ, Yin XW, Liu HH, Du WX, Shi LY, Huang YB, Wang F, Liu CF, Cao YJ and Zhang YL: Rapamycin inhibits ox-LDL-induced inflammation in human endothelial cells in vitro by inhibiting the mTORC2/PKC/c-Fos pathway. Acta Pharmacol Sin 39: 336-344, 2018.

44. Evans TD, Jeong SJ, Zhang X, Sergin I and Razani B: TFEB and trehalose drive the macrophage autophagy-lysosome system to protect against atherosclerosis. Autophagy 14: 724-726, 2018.

45. Michiels CF, Kurdi A, Timmermans JP, De Meyer GRY and Martinet W: Spermidine reduces lipid accumulation and necrotic core formation in atherosclerotic plaques via induction of autophagy. Atherosclerosis 251: 319-327, 2016.

46. Liu K and Czaja MJ: Regulation of lipid stores and metabolism by lipophagy. Cell Death Differ 20: 3-11, 2013.

47. Zhu X, Xiong T, Liu P, Guo X, Xiao L, Zhou F, Tang Y and Yao P: Quercetin ameliorates HFD-induced NAFLD by promoting hepatic VLDL assembly and lipophagy via the IRE1a/XBP1s pathway. Food Chem Toxicol 114: 52-60, 2018.

48. Leng S, Iwanowycz S, Saaoud F, Wang J, Wang Y, Sergin I, Razani B and Fan D: Ursolic acid enhances macrophage autophagy and attenuates atherogenesis. J Lipid Res 57: 1006-1016, 2016.

This work is licensed under a Creative Commons Attribution-NonCommercial-NoDerivatives 4.0 International (CC BY-NC-ND 4.0) License. 